\title{
SC1, an immunoglobulin-superfamily cell adhesion molecule, is involved in the brain metastatic activity of lung cancer cells
}

\author{
YUKA KUBOTA, NAOKI KIRIMURA, HATSUKI SHIBA, KAZUHIDE ADACHI and YASUHIRO TSUKAMOTO \\ Department of Animal Hygiene, Graduate School of Environmental and Biological Sciences, \\ Kyoto Prefecture University, Shimogamo, Kyoto 606-8522, Japan
}

Received September 9, 2014; Accepted June 5, 2015

DOI: $10.3892 / \mathrm{ol} .2015 .3576$

\begin{abstract}
SC1 is a cell adhesion molecule that belongs to the immunoglobulin superfamily; this molecule was initially purified from the chick embryonic nervous system and was reported to exhibit homophilic adhesion activity. SC1 is transiently expressed in various organs during development and has been identified in numerous neoplastic tissues, including lung cancer and colorectal carcinomas. The present study focused on the encephalic metastasis of lung cancer cells with respect to the potential function of $\mathrm{SC}$, as this molecule is known to be consistently expressed in the central nervous system as well as lung cancers. SC1 complementary DNA was introduced into A549 cells, a human lung cancer-derived cell line. The stable overexpression of the $\mathrm{SC1}$ protein in A549 cells was demonstrated to enhance the self-aggregation of the cells. In addition, the SC1 transfectants enhanced the metastatic and invasive potential to the encephalic parenchyma following implantation into nude mice. In conclusion, the results of the present study demonstrated that cell adhesion due interactions between SC1 on brain tissue and $\mathrm{SC} 1$ on lung cancer cells was involved in the malignant aspects of lung cancer, including invasion and metastasis to the brain.
\end{abstract}

\section{Introduction}

Numerous studies have confirmed that cell adhesion molecules (CAMs) are essential for normal histogenesis as well as tumor malignancies (1-3). In addition, it was reported that dysregulated CAM expression was often involved in certain tumor behavior $(4,5)$. Of note, the inhibition or attenuation of E-cadherin expression in epithelial cells was demonstrated to enhance cellular transformation. A certain

Correspondence to: Professor Yasuhiro Tsukamoto, Department of Animal Hygiene, Graduate School of Environmental and Biological Sciences, Kyoto Prefecture University, 1-5 Hangi-cho, Shimogamo, Kyoto 606-8522, Japan

E-mail: ytsuka@kpu.ac.jp

Key words: lung cancer, SC1, metastasis, cell adhesion molecule
CAM, deleted in colorectal cancer (DCC), is a member of the immunoglobulin (Ig) superfamily, which is commonly lost during the development of colorectal tumors. The loss of expression of CAMs, such as DCC, has therefore been suggested to enable the migration and escape of tumor cells from the primary lesion, subsequently resulting in the invasion and the dissemination of metastases. By contrast, various other CAMs have been reported to be overexpressed in numerous types of tumors, which may promote tumor cell invasion and metastasis through their adhesive activities $(4,6)$.

$\mathrm{SC} 1$ is a cell adhesion molecule that belongs to the Ig superfamily; this molecule was identified in several independent studies, each of which gave it a different title, $\mathrm{SC} 1$, bursal epithelium and neurons or DM-general receptor for phosphoinositides 1-associated scaffold protein (7-10). This protein possesses an intracellular domain that carries three C2-type Ig-like motifs followed by two V-type motifs. The homophilic properties of SC1 have been revealed using cellular adhesion experiments on substrates and self-aggregation assays in vitro $(8,11-13)$. By contrast, previous studies have also reported heterophilic interactions of $\mathrm{SC} 1$, such as that with neuron-glia ( $\mathrm{Ng})$-CAM during the extension of neurites from sympathetic neurons as well as that with CD6 in the hematopoietic system $(14,15)$. SC1 homologs have been confirmed in zebrafish, mice, rats and humans (16). During embryogenesis in chicks, SC1 is transiently expressed, most notably in nervous and hematopoietic system cells as well as the cells of certain epithelia $(9,12)$. In addition, SC1 was reported to have roles in a variety of physiological processes, which include hematopoiesis, thymic development, immune responses, neurite extension, migration of neural cells and osteogenesis.

The human homolog of SC1, activated leukocyte CAM/CD166/MEMD, was reported to be expressed in malignant melanoma cells and represents a novel molecular marker for the progression of melanoma, which has potential prognostic value $(17,18)$. Previous studies have revealed that SC1 promoted the metastatic activity of mammary gland tumor and lymphoma cells; in addition, the hemophilic binding ability of SC1 enhances the interaction of tumor cells with blood vessels (19-21). Evidence was also found which indicated that SC1 was strongly expressed in sporadic cases of encephalic metastasis of human breast cancer (22). 
A549 cells, a lung cancer cell line derived from adenocarcinoma, were found to be almost negative for $\mathrm{SC}$, which led to the hypothesis that this cell line was a good model for investigating the functions of SC1 in the brain metastasis of lung cancer. In the present study, SC1-transfected A549 cells were established and implanted into nude mice in order to investigate the involvement of SC1 in the metastasis of lung cancer to the brain.

\section{Materials and methods}

Establishment of SC1-expressing A549 cells by gene introduction. A549 cells (Riken BioResource Center, Ibaraki, Japan) were cultured with Dulbecco's modified Eagle's medium (DMEM; Wako Pure Chemical Industries Ltd., Osaka, Japan) containing $10 \%$ fetal calf serum (Wako Pure Chemical Industries Ltd.) at $37^{\circ} \mathrm{C}$. Semiconfluent cells $(\sim 80 \%)$ were transfected with the pcDNA 3.1 plasmid vector (Invitrogen Life Technologies, Carlsbad, CA, USA) containing the intact human SC1 gene. In addition, parental A549 cells were transfected with an empty vector in order to produce mock-transfected cells. These transfectants were cultured in DMEM containing $10 \mu \mathrm{g}$ G418 (Gibco-BRL, Carlsbad, CA, USA) and then the resistant colonies were selected and further cultured. Furthermore, stable SC1- and mock-transfected clones were established following confirmation by SC1 immunocytochemistry, as described below; these cells were used for all subsequent experiments.

Immunocytochemistry. Each of the transfectants were grown on culture plates at $37^{\circ} \mathrm{C}$ in an atmosphere of $5 \% \mathrm{CO}_{2}$ for 2 days and then fixed in Zamboni's solution (Wako Pure Chemical Industries Ltd.) at semiconfluent stages. The cultures were washed twice with phosphate-buffered saline (PBS; Wako Pure Chemical Industries Ltd.) and incubated with a monoclonal mouse antibody against the SC1 protein (1:500; Department of Animal Hygiene, Kyoto Prefecture University, Kyoto, Japan) for $1 \mathrm{~h}$ at $37^{\circ} \mathrm{C}$. Following being washed twice with PBS, the cells on culture plates were incubated with Alexa Fluor-conjugated goat anti-mouse IgG (1:500; cat. no. 150117; Abcam, Tokyo, Japan) for $1 \mathrm{~h}$ at $37^{\circ} \mathrm{C}$. Following sufficient washes with $\mathrm{PBS}$, the cells were examined under a fluorescent microscope (Eclipse E600; Nikon Corporation, Tokyo, Japan).

In vitro cell aggregation assay. Each of the transfectants on culture dishes was dispersed with a solution containing $0.05 \%$ trypsin and $0.01 \mathrm{mM}$ EDTA (Wako Pure Chemical Industries Ltd.); subsequently, cells were collected via centrifugation for $10 \mathrm{~min}$ at $400 \mathrm{x}$ g. Cells were then resuspended in DMEM at a density of $9 \times 10^{6}$ cells $/ \mathrm{ml}$. Each cell suspension $(0.5 \mathrm{ml})$ was incubated in an Eppendorf tube at $37^{\circ} \mathrm{C}$ with or without anti-SC1 monoclonal antibody (75 $\mu \mathrm{g} / \mathrm{ml}$; Department of Animal Hygiene, Kyoto Prefecture University). Following $1 \mathrm{~h}$ of incubation, the tubes were inverted once and the cell suspensions were observed under a microscope (Eclipse TS100; Nikon Corporation).

Implantation of SC1-transfected cells into the cerebrum of nude mice. The SC1-transfectants suspended in DMEM $\left(2 \times 10^{7}\right.$ cells $\left./ \mathrm{ml}\right)$ were injected into the temporal lobe medulla of 7-week-old female nude mice ( $\mathrm{n}=5$; Shimizu Laboratory Supplies Co. Ltd., Kyoto, Japan) under deep general anesthesia with pentobarbital sodium solution $(64.8 \mathrm{mg} / \mathrm{ml}$; Kyoritsu Seiyaku, Tokyo, Japan). All animals were housed individually in plastic cages at controlled temperatures $\left(22 \pm 1^{\circ} \mathrm{C}\right)$ and relative humidity $(55 \pm 10 \%)$ and exposed to $12 \mathrm{~h}$ light/ dark cycles with free access to water and commercial feed (Shimizu Laboratory Supplies Co. Ltd.). Other SC1-transfectants were pre-incubated with anti-SC1 monoclonal antibody (75 $\mu \mathrm{g} / \mathrm{ml}$; Department of Animal Hygiene, Kyoto Prefecture University) for $2 \mathrm{~h}$ at $37^{\circ} \mathrm{C}$ and subsequently injected into a group of nude mice $(n=5)$. At 8 days following cell injections, all animals were sacrificed through an overdose of pentobarbital sodium solution $(64.8 \mathrm{mg} / \mathrm{ml}$; Kyoritsu Seiyaku) and the brains were removed and fixed with formalin solution (Wako Pure Chemical Industries Ltd.) for histopathology studies. All of the animal experiments were performed in accordance with the guidelines for studies with laboratory animals of the Kyoto Prefectural University Experimental Animal Committee (Kyoto, Japan).

Histopathology. The temporal lobes of mice were embedded in paraffin (Wako Pure Chemical Industries Ltd.) and cut into $3-\mu \mathrm{m}$ sections with a microtome and were subsequently stained with hematoxylin and eosin, according to the routine procedure (19). The sections were then observed under a light microscope (Eclipse E600; Nikon Corporation).

\section{Results}

Expression of SC1 proteins in transfectants. The expression of SC1 in A549 lung cancer cells was evaluated through immunocytochemical analyses using an anti-SC1 antibody. $\mathrm{SC} 1$ protein expression was not identified in either the parental cells or mock-transfected cell lines. By contrast, SC1 transfectants strongly expressed SC1 proteins in the membrane (Fig. 1). All of the SC1-transfected cells stably expressed SC1 on their cell surface even following further passages. Therefore, it was confirmed that the established transfectants stably expressed $\mathrm{SC1}$ and these SC1-expresssing transfectants were used for all subsequent experiments.

In vitro cell aggregation assay. An in vitro cell aggregation assay revealed that cell aggregation was markedly increased in SC1-transfected cells compared with that of the mock-transfected cells (Fig. 2A and B). Of note, the SC1-transfected cells began to self-aggregate from the initiation of incubation, which resulted in the formation of cell clusters; by contrast, mock-transfected cells demonstrated only slight aggregation. In addition, following co-incubation of the SC1 transfectant cells with an anti-SC1 antibody, the cells only exhibited slight aggregation (Fig. 2C). These results therefore indicated that SC1 expression in A549 cells was functional with respect to the cell-cell interactions; in addition, it was revealed that the anti-SC1 antibody was able to inhibit this cell aggregation activity.

Implantation of SC1-transfected cells into the cerebrum of nude mice. A549 cells transfected with SC1 with or without pre-incubation with anti-SC1 antibodies were injected into 

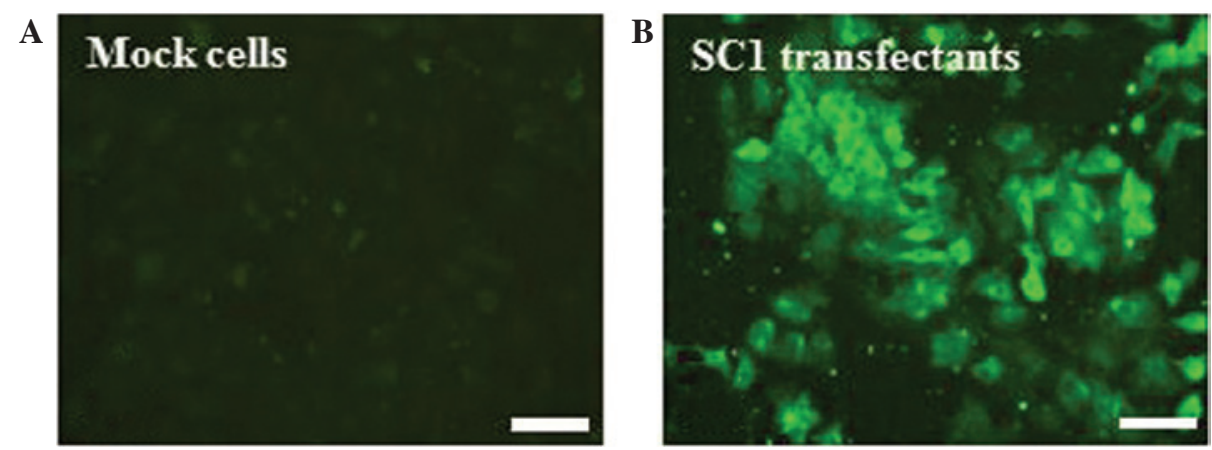

Figure 1. Expression of SC1 protein in A549 lung cancer cells. The expression levels of SC1 in (A) mock- and (B) SC1-transfected A549 cells were examined immunocytochemically under a fluorescent microscope. Specific immunofluorescence was not observed in mock transfectants, whereas SC1 transfectants exhibited strong signals. Scale bars, $25 \mu \mathrm{m}$.
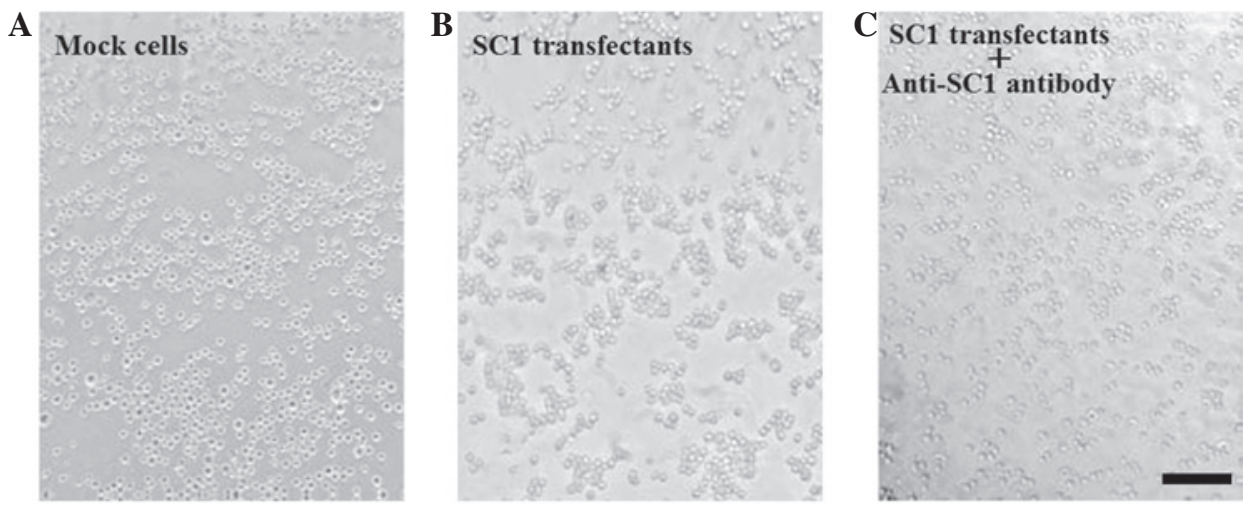

Figure 2. In vitro cell aggregation assays. An aliquot of mock- and SC1-transfectants was incubated for $1 \mathrm{~h}$ at $37^{\circ} \mathrm{C}$, then cell particles were observed under a light microscope (Eclipse TS100; Nikon Corporation). (A) Mock transfectants demonstrated only slight cell aggregation activity. By contrast, (B) SC1 transfectants aggregated with each other and formed cell clusters following $1 \mathrm{~h}$ of incubation. (C) An anti-SC1 antibody inhibited the cell aggregation activity of SC1 transfectants, these cells demonstrated only slight aggregation. Thus, the SC1 protein expressed on A549 lung cancer cells was functional with respect to cell-cell binding. Scale bar, $100 \mu \mathrm{m}$.
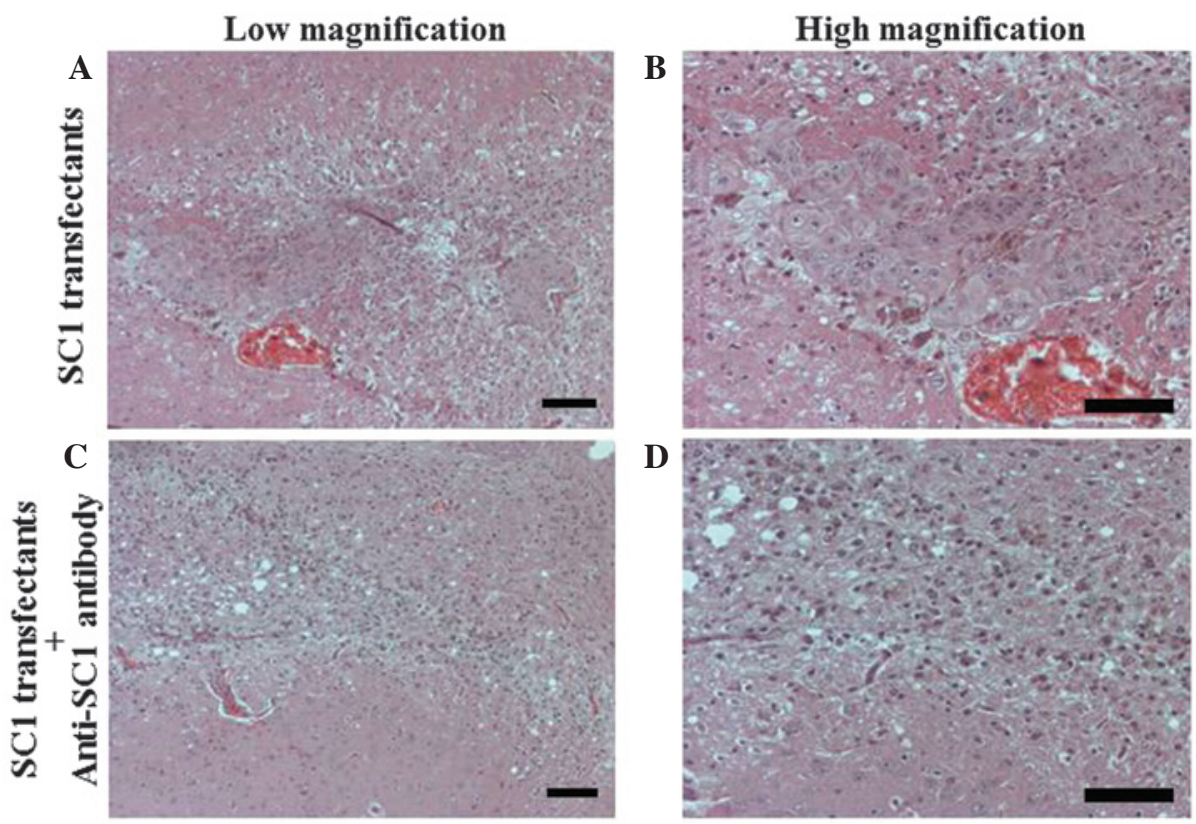

D

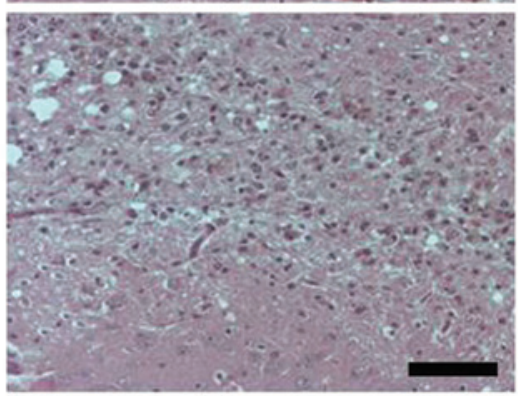

Figure 3. Histopathology of nude mice brains implanted with SC1 transfectants. Paraffin sections of nude mice cerebrums engrafted with SC1 transfectants were cut and stained with hematoxylin and eosin, and observed under a light microscope. (A and B) In the medulla of the temporal lobes of nude mice implanted with SC1 transfectants, severe metastatic legions were observed. Adenocarcinomatous tumors grew invasively and colliquative necrosis, demyelination, inflammation and hemorrhage were observed. (C and D) SC1 transfectants pre-incubated with anti-SC1 antibodies prior to implantation demonstrated only slight pathological legions with limited colliquative necrosis, demyelination and inflammation; the invasive growth of adenocarcinoma was not detectable. Accordingly, it was suggested that SC1 enhanced the malignant potential of A549 lung cancer cells in the mouse brain. Scale bars, $200 \mu \mathrm{m}$. Low magnification, x25. High magnification, x100. 
the temporal lobe of nude mice. The metastatic activities of cells were then examined histopathologically. As shown in Fig. 3A and B, in all mice injected with SC1 transfectants, severe metastatic legions were observed in the medulla of the temporal lobe; in addition, adenocarcinomatous tumors grew invasively in the brain matter and colliquative necrosis, demyelination, inflammation and hemorrhage were also prominent in the areas surrounding the metastatic tissue. By contrast, the SC1 transfectants incubated with the anti-SC1 antibody demonstrated only slight lesions in all mice brains, in which slight colliquative necrosis, demyelination and inflammation were observed, although the invasive growth of cells was not detectable (Fig. 3C and D). These results therefore indicated that the anti-SC1 antibodies inhibited the metastatic and invasive activities of SC1-transfected cells in the mouse brain. Therefore, it was suggested that the A549 cells acquired their high metastatic and invasive potential as a result of the $\mathrm{SC} 1$ expression on the cell membrane.

\section{Discussion}

Lung cancer is the primary cause of cancer-associated mortality in males and females worldwide. Patients suffering from lung adenocarcinoma are commonly diagnosed with early and distal metastasis to the brain and bones, which results in mortality (23). In the present study, SC1-expressing A549 cells were established through transfecting parental A549 cells with SC1 complementary DNA. The role of SC1 in the metastatic and invasive potential of lung cancer cells was subsequently investigated in the brains of nude mice injected with these cells. The results confirmed the functional ability of the SC1 proteins expressed by transfected cells in vitro in regards to cell-cell binding, as demonstrated using cell aggregation assays. Of note, the results of the present study revealed that $\mathrm{SC} 1$ was able to markedly promote the metastatic and invasive potential of A549 cells in mice models in vivo. However, preliminary studies demonstrated that there were no marked differences in the doubling times among the cells incubated with and without anti-SC1 antibodies (data not shown); therefore, the enhanced metastasis of SC1-transfectants in the mouse brain may have occurred due to an increase in cell adhesion among the tumor cells or tumor-brain cell interactions, including those among nerve cells, glial cells and blood vessels. SC1 has been reported to be enriched in normal tissues and certain neoplastic tissues $(8,19-21)$. These distribution patterns of SC1 suggest that SC1-transfected cells have the ability to attach to the surface of encephalic parenchyma cells following SC1-SC1 binding.

It has been well-established that in situ metastasis is the result of a multistep process, involving an altered association between cancer cells and normal tissues. The initial step requires the detachment of cancer cells from the primary tumor sites, followed by intravasation through the neighboring cells and/or networks of the extracellular matrix networks; subsequently, cancer cells must interact with the blood vessels and extravasate into the metastatic sites (24). E-cadherin expression inhibition or dysregulation was suggested to be important for the release of cells from the primary tumor site, as it results in reduced contact-mediated regulation $(24,25)$. In addition, the upregulation of certain other CAMs promotes cell migration through the extracellular matrix (4). Interactions of SC1 have been identified with itself, as well as with Ng-CAM and CD6 $(14,15)$; however, it is likely that SC1 also interacts with other unidentified proteins. These SC1-mediated cell adhesion interactions may lead to the enhanced metastatic activity observed in A549 cells. However, the underlying pathways of SC1-mediated increases in tumor cell invasion and metastasis appear to be complex.

In conclusion, the present study revealed that the expression of SC1 promoted the encephalic invasive activity of A549 cells as a result of its increased adhesive activity. Future studies are required in order to examine the role of $\mathrm{SC} 1$ in the blood-borne metastasis of cancer from the lungs to the encephalon. It was hypothesized that $\mathrm{SC} 1$ has a role in the invasion of lung cancer cells into the encephalic parenchyma as well as in blood-borne metastasis. Therefore, the identification of the role of $\mathrm{SC} 1$ in various metastatic systems may potentially inhibit the SC1-dependent aspects of the malignant progression of cancers.

\section{References}

1. Edelman GM: Cell adhesion and the molecular processes of morphogenesis. A Rev Biochem 54: 135-169, 1983.

2. Klymkowsky MW and Parr B: The body language of cells: The intimate connection between cell adhesion and behavior. Cell 83: 5-8, 1995.

3. Obrink B: Epithelial cell adhesion molecules. Exp Cell Res 163: $1-21,1986$.

4. Albelda SM: Role of integrins and other cell adhesion molecules in tumor progression and metastasis. Lab Invest 68: 4-17, 1993;

5. Takeichi M: Cadherins in cancer; implications for invasion and metastasis. Curr Opini Cell Biol 5: 806-811, 1993.

6. Tsukamoto Y, Taira E, Miki N and Sasaki F: The role of gicerin, a novel cell adhesion molecule, in development, regeneration and neoplasia. Histol Histopathol 16: 563-571, 2001.

7. Tanaka $\mathrm{H}$ and Obata K: Developmental changes in unique cell surface antigens of chick embryo spinal motorneurons and ganglion cells. Dev Biol 106: 26-37, 1984.

8. Tanaka H, Matsui T, Agata A, Tomura M, Kubota I, McFarland KC, Kohr B, Lee A, Phillips HS and Shelton DL: Molecular cloning of and expression of a novel adhesion molecule, SC1. Neuron 7: 535-545, 1991.

9. Pourquié O, Corbel C, Le Caer JP, Rossier J and Le Douarin NM: BEN, a surface glycoprotein of the immunoglobulin superfamily, is expressed in a variety of developing systems. Proc Nat Acad Sci USA 89: 5261-5265, 1992.

10. Burns FR, von Kannen S, Guy L, Raper JA, Kamholz J and Chang S: DM-GRASP, a novel immunoglobulin suprerfamily axonal surface protein that supports neurite extension. Neuron 7: 209-220, 1991.

11. el-Deeb S, Thompson SC and Covault J: Characterization of a cell surface adhesion molecule expressed by a subset of developing chick neurons. Dev Biol 149: 213-227, 1992.

12. Corbel C, Pourquié O, Cormier F, Vaigot $P$ and Le Douarin NM: BEN/SC1/DM-GRASP, a homophilic adhesion molecule, is required for in vitro myeloid formation by avian hemopoietic progenitors. Proc Nat Acad Sci USA 93: 2844-2849, 1996.

13. DeBernardo AP and Chang S: Native and recombinant DM-GRASP selectively support neurite extension from neurons that express GRASP. Dev Biol 169: 65-75, 1995.

14. DeBernardo AP and Chang S: Heterophilic interactions of DM-GRASP: Grasp-NgCAM interactions involved in neurite extension. J Cell Biol 133: 657-666, 1996.

15. Bowen MA, Patel DD, Li X, et al: Cloning, mapping and characterization of activated leukocyte-cell adhesion molecule (ALCAM), a CD6 ligand. J Exp Med 181: 2213-2220, 1995.

16. Swart GW: Activated leukocyte cell adhesion molecule (CD166/ALCAM): Developmental and mechanistic aspects of cell clustering and cell migration. Eur J Cell Biol 81: 313-321, 2002. 
17. van Kempen LC, van den Oord JJ, van Muijen GN, Weidle UH, Bloemers HP and Swart GW: Activated leukocyte cell adhesion molecule, $\mathrm{CD} / 166$, a marker of tumor progression in primary malignant melanoma of the skin. Am J Pathol 156: 769-774, 2000.

18. van Kempen LC, Meier F, Egeblad M, Kersten-Niessen MJ Garbe C, Weidle UH, Van Muijen GN, Herlyn M, Bloemers HP and Swart GW: Truncation of activated leukocyte cell adhesion molecule: A gateway to melanoma metastasis. J Invest Dermatol 122: 1293-1301, 2004.

19. Tsukamoto Y, Namikawa T, Tatesaki R, Kotani T and Tanaka H: Expression and adhesive activity of $\mathrm{SC} 1$, an Ig superfamily cell adhesion molecule, in sporadic nephroblastomas of chicken. Oncol Rep 15: 137-141, 2006.

20. Adachi K, Hagimori K, Kato H, Fukuda K, Kikuta M and Tsukamoto Y: Potential role of SC1, a cell adhesion molecule, in mammary gland tumors. Mol Med Rep 1: 219-224, 2008.

21. Handharyani E, Tsukamoto $M$ and Tsukamoto $Y$ : Expression of SC1, a cell adhesion molecule, promotes the metastatic activities of the Gallus gallus lymphoblastoid cell line MDCC-MSB1 derived from Marek's disease. Avian Pathol 40: 111-115, 2011.
22. Ihnen M, Kilic E, Köhler N, et al: Protein expression analysis of ALCAM and CEACAM6 in breast cancer metastases reveals significantly increased ALCAM expression in metastases of the skin. J Clin Pathol 64: 146-152, 2011.

23. Tsai CM, Yen GC, Sun FM, Yang SF and Weng CJ: Assessment of the anti-invasion potential and mechanism of select cinnamic acid derivatives on human lung adenocarcinoma cells. Mol Pharm 10: 1890-1900, 2013

24. Cunningham BA: Cell adhesion molecules as morphoregulators. Curr Opin Cell Biol 7: 628-633, 1995.

25. Takeichi M: Cadherins in cancer: Implications for invasion and metastasis. Curr Opin Cell Biol 5: 806-811, 1993. 